\title{
Recycling the ISM of Starburst galaxies: The SN heating efficiency paradigm
}

\author{
Claudio Melioli ${ }^{1}$ and E. M. de Gouveia Dal Pino ${ }^{1}$ \\ ${ }^{1}$ IAG-USP, Cidade Universitaria, Rua do Matão, 1226, 05508-090, São Paulo, SP, Brazil \\ email: cmelioli@astro.iag.usp.br, dalpino@astro.iag.usp.br
}

\begin{abstract}
We present a semi-analytic model able to trace the thermalisation history of the ISM in a Starburst region and determine the SN heating efficiency evolution.
\end{abstract}

The interstellar medium heated by supernova explosions (SN) may acquire an expansion velocity larger than the escape velocity and leave the galaxy through a supersonic wind. Galactic winds are effectively observed in many local starburst galaxies (see, e.g., Lehnert \& Heckman 1996). SN ejecta are transported out of the galaxies by such winds which thus affect the chemical evolution of the galaxies. The effectiveness of the processes mentioned above depends on the heating efficiency (HE) of the SNe, i.e. on the fraction of the SN explosion energy that remains effectively stored in the ISM gas and is not radiated away. The value of $\mathrm{HE}$, in particular in starburst (SB) galaxies, is a matter of debate. Larson (1974) evaluates that the energy which is effectively transmitted to the gas is $\sim 10 \%$ of the explosion energy. This value of HE has been adopted in a number of SB models (e.g. Murakami \& Babul 1999). Bradamante, Matteucci \& D'Ercole (1998), following a different line to estimate $\mathrm{HE}$, find $\sim 0.03$ in the cases of interest, and this value has been adopted by Ferrara \& Tolstoy (2000) and by Recchi, Matteucci \& D'Ercole (2001) in their models of dwarf galaxies. These low values of HE have been questioned by several authors. Following Cox \& Smith (1974), Larson (1974) warned that, for a high SN rate, the collisions of the first generation of SNRs possibly lead to the formation of an interconnected network of tunnels containing very hot, tenuous gas, maintained at high temperature by continuing supernova explosions. The successive generations of SNRs are likely to produce a value of $\mathrm{HE}$ close to unity. In fact, the remnants do not reach high densities during their expansion, and their radiative losses are expected to remain negligible as the emissivity is proportional to the square of the density. Strickland \& Stevens (2000) argue that with these low values of HE the galactic wind observed in M82 (the best studied SB with a galactic wind) could be hardly driven. Chevalier \& Clegg (1985) find that $100 \%$ is a likely value for HE in M82.

In order to try to put some light on this debate, we have constructed a simple semianalytic model, considering the essential ingredients of a SB environment which is able to qualitatively trace the thermalisation history of the ISM in a SB region and determine HE evolution. Our study has also been accompanied by fully $3-\mathrm{D}$ radiative cooling, hydrodynamical simulations of SNR-SNR and SNR-clouds interactions (see Melioli, de Gouveia Dal Pino \& Raga 2004a, and these proceedings). We find that, as long as the typical time scale of mass-loss of the clouds to the ambient medium, which is often dominated by photoevaporation, remains shorter than the time scale at which the SNRs interact to form a superbubble, the SN heating efficiency remains very small, as radiative cooling of the gas dominates. If there is a continuous production of clouds by the gas swept by the SNR shells, this occurs during the first $\leqslant 16$ Myrs of the SB activity (which 

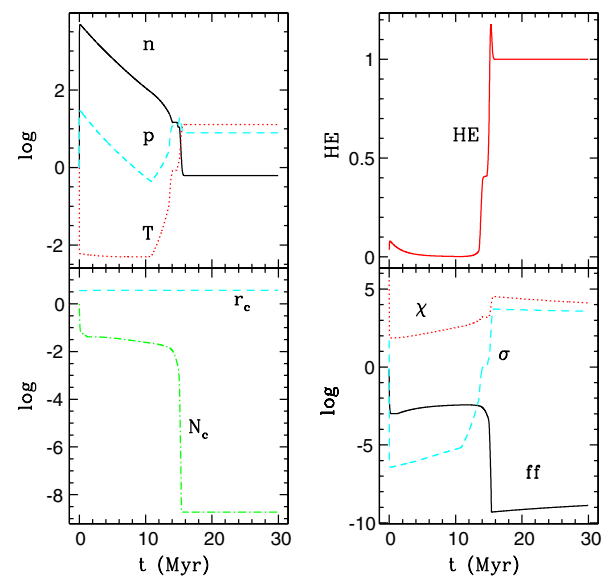

Figure 1. Ambient density $(n)$, temperature $(T)$ and pressure $(p)$ evolution in the top-left panel for a spherical SB with initial $M_{b}=10^{6} \mathrm{M}_{\odot}, R_{S B}=100 \mathrm{pc}, n_{0}=0.01 \mathrm{~cm}^{-3}, T_{0}=2 \times 10^{6}$ $\mathrm{K}, p_{0}=6.35 \times 10^{-12} \mathrm{dy} \mathrm{cm}^{-2}$ and total gas mass $M_{g}=8 \times 10^{6} M_{\odot}$. In the bottom-left panel: number of clouds $\left(N_{c}\right)$ and radius of clouds $\left(r_{c}\right)$ evolution; in the top-right panel: the SN heating efficiency (HE); and in the bottom-right panel: the clouds filling factor, $\left(f f=N_{c} \mathcal{V}_{c} / V_{S B}\right.$, where $\mathcal{V}_{c}$ is the volume of a single cloud), the saturation parameter ( $\sigma$, eq. 15) and the cloud to ambient gas density ratio $\left(\chi=\rho_{c} / \rho\right)$. The ambient density, temperature and pressure, and the number and radius of clouds are normalized to their initial values (in $\log$ scale). The initial radius, temperature and density assumed for the clouds are $r_{c}=6.5 \times 10^{-3} \mathrm{pc}, T_{c}=100 \mathrm{~K}$, and $n_{c}=6 \times 10^{5} \mathrm{~cm}^{-3}$, respectively (from Melioli \& de Gouveia Dal Pino 2004).

lasts $\sim 30$ Myrs), after which the efficiency rapidly increases to one, leading to a possible galactic wind formation (see Figure 1).

Under an extreme condition in which no clouds are allowed to form, other than those that were already initially present in the SB environment, then in this case HE increases to one in only few Myrs.

We therefore conclude that the $\mathrm{HE}$ value has a time-dependent trend that is sensitive to the initial conditions of the system and cannot be simply assumed to be $\sim 1$, as it is commonly done in most SB galactic wind models. We also should notice that studies that search for a potential connection between SBs and AGN activity, may be favored by the results of the present analysis as these predict a delay in the expulsion of gas from the SB. The gas retained in the system may lead to new bursts of star formation and increasing deposition of material in the center of the SB that, in turn, may eventually form a BH (see Melioli \& de Gouveia Dal Pino 2004).

\section{References}

Bradamante, F., Matteucci, F., \& D'Ercole, A. 1998, A\&A, 337, 338

Chevalier, R. A., \& Clegg, A. W. 1985, Nature, 317, 44

Cox, D. P., \& Smith, B. W. 1974, ApJ, 189, L105

Ferrara, A., \& Tolstoy, E. 2000, MNRAS, 313, 291

Larson, R. B. 1974, MNRAS, 169, 229

Lehnert, M. D., \& Heckman, T. M. 1996, ApJ, 462, 651

Melioli, C., \& de Gouveia Dal Pino, E. M. 2004, A\&A, in press

Melioli, C., de Gouveia Dal Pino, E. M., \& Raga, A. C. 2004a, in preparation

Murakami, I., \& Babul, A. 1999, MNRAS, 309, 161

Recchi, S., Matteucci, F., \& D'Ercole, A. 2001, MNRAS, 322, 800

Strickland, D. K., \& Stevens, I. R. 2000, MNRAS, 314, 511 